\title{
IMPACTO DE UN ESTILO DE VIDA ACTIVO SOBRE LA MEMORIA EMOCIONAL
}

\author{
IMPACT OF AN ACTIVE LIFE-STYLE ON \\ EMOTIONAL MEMORY
}

MAXIMILIANO BOSSIO ${ }^{1}$ Y NADIA JUSTEL ${ }^{2}$

Cómo referenciar este artículo/How to reference this article:

Bossio, M. y Justel, N. (2018). Impacto de un estilo de vida activo sobre la memoria emocional [Impact of an active life-style on emotional memory]. Acción Psicológica, 15(1), 39-56. https://doi.org/10.5944/ap.15.1.21446

\section{Resumen}

El ejercicio aeróbico y de resistencia se ha relacionado con la promoción de la salud física, además en los últimos años se han investigado sus beneficios a nivel cognitivo. En base a ello, el objetivo de este trabajo fue evaluar el posible efecto de tener un estilo de vida activo sobre la memoria emocional. Participaron del estudio 48 adultos de edad temprana y media (rango: 18-58 años) que llevaron a cabo un auto-reporte mediante el Cuestionario Internacional de Actividad Física dando cuenta de la cantidad y tipo de actividad física realizada en una semana habitual o típica, es decir aquella sostenida durante los últimos tres meses de sus vidas. Para evaluar la memoria emocional los participantes observaron una serie de imágenes positivas, negativas y neutras seleccionadas del Sistema Internacional de Imágenes Afectivas. Luego se evaluó cuántas imágenes recordaban y reconocían (tanto de modo inmediato como diferido). Se halló que los sujetos que poseían un nivel alto de actividad física en su vida diaria recordaban inmediatamente más estímulos de valencia positiva que aquellos con niveles moderados y bajos. Además, este grupo obtuvo mejores resultados en la evaluación de reconocimiento inmediato. Por tanto, incorporar a la actividad física y deportiva como un estilo de vida, mostraría tener efectos favorables en la memoria de estímulos visuales vinculados a emociones positivas.

Correspondencia: Nadia Justel. Lab. Interdisciplinario de Neurociencia Cognitiva LINC CEMSC3 ECyT UNSAM. CONICET.

Email: nadiajustel@conicet.gov.ar

ORCID: Maximiliano Bossio (http://orcid.org/0000-0002-0380-3525) y Nadia Justel (http://orcid.org/0000-0002-01453357)

${ }^{1}$ Universidad de Buenos Aires, Argentina.

${ }^{2}$ CONICET, Argentina

Recibido: 28 de febrero de 2018 .

Aceptado: 14 de mayo de 2018. 
Palabras clave: Actividad deportiva; Memoria; Emociones; Modulación.

\begin{abstract}
Aerobic and resistance exercise has been related to physical health promotion, besides in the last years its effects at a cognitive level has been investigated. Based on that, the aim of the current work was to evaluate the possible effect of having an active life style on emotional memory. Fortyeight adults of early and middle age participated (range: 18-58 years old) who complete a report through the International Physical Activity Questionnaire about the amount and type of physical activity performed in a typical or regular week, i.e. a week sustained in the last three months of their lives. To evaluate emotional memory participants watched positive, negative and neutral images selected from the International Affective Picture System. Then, how many images they recall and recognize was evaluated (immediate and deferred measures). Participants that had a high level of physical activity in their lives remember immediately more positive images than those participants with a low or moderate level of activity. Besides, this group obtained better results in the immediate recognition measure. Thus, incorporate physical activity as a life style shows benefits in the emotional positive memory.
\end{abstract}

Keywords: Sport activity; Memory; Emotions; Modulation.

\section{Introducción}

En los últimos años aumentó el interés de los investigadores en relación a la actividad aeróbica y deportiva, ya que modularía las funciones cognitivas tanto en animales como humanos (Erickson et al., 2011; Hogan, Mata y Carstensen, 2013; Ratey y Loehr, 2011). Esta nueva perspectiva brinda un valor agregado al ejercicio aeróbico y de resistencia, que por décadas ha sido relacionado principalmente con la promoción de la salud, el fortalecimiento de las estructuras musculoesqueléticas del cuerpo, mejoras en los niveles de presión sanguínea, y en la salud cardiovas- cular global (Hogan et al., 2013). Además de estas mejoras físicas, en la última década varios estudios señalaron que se sumarían beneficios a nivel neurocognitivo (Chang et al., 2014; Labelle, Bosquet, Mekary y Bherer, 2013; Wengaard, Kristoffersen, Harris y Gundersen, 2017). Se ha descubierto que la utilización de dosis agudas de ejercicio, entendido como una exposición que no supera la hora de trabajo y sucede en un único momento y lugar específico, así como también el haber realizado deporte a lo largo de la vida genera un efecto beneficioso sobre las funciones cognitivas en diversas poblaciones (Chen, Yan, Yin, Pan y Chang, 2014; Hayes, Forman y Verfaellie, 2014; Hwang et al., 2016; Kimura y Hozumi, 2012; Lee et al., 2014; Soga, Shishido y Nagatomi, 2015; Wengaard et al., 2017). Por lo que el ejercicio físico está emergiendo como una estrategia prometedora de bajo costo que mejoraría las funciones cognitivas, sería accesible a la mayoría de la población y prácticamente no tendría efectos secundarios.

Se ha encontrado que el hipocampo y regiones temporales son mayores en los sujetos que poseen un nivel cardiorrespiratorio más desarrollado fruto del entrenamiento aeróbico cardíaco (Erickson et al., 2009; Honea et al., 2009). Estas estructuras son relevantes para la formación y consolidación de memorias, y en relación a ello se halló que realizar ejercicio físico mejora el aprendizaje y la memoria, aumenta la proliferación celular y la sobrevida del hipocampo, e incrementa el volumen de materia gris y blanca (Colcombe et al., 2006; van Praag et al., 2005). Diferentes investigaciones que trabajaron con niños (Jäger, Schmidt, Conzelmann y Roebers, 2015) y adultos (Hayes et al., 2014) descubrieron que los sujetos que poseían mayor nivel de involucramiento con la actividad física deportiva eran los que obtenían mejores resultados en evaluaciones con demanda cognitiva en memoria de trabajo, así como memoria espacial (Erickson et al., 2011).

Específicamente en relación a la memoria, a lo largo de los años se propusieron múltiples clasificaciones sobre los sistemas de memoria, ninguna de las cuales podría ser considerada actualmente totalmente satisfactoria (Ruetti, Justel y Bentosela, 2009). Una de las clasificaciones mayormente aceptadas es aquella que distingue entre memoria explicita e implícita. Dentro de la primera se encontraría la memoria episódica y semántica; mientras que la memoria implícita hace referencia al aprendizaje de habilida- 
des (Squire, 1987; Tulving, 2002). Por otro lado, la memoria emocional podría considerarse una subdivisión de la memoria explícita y es el resultado del almacenamiento de la información que estuvo acompañada por factores de alarma o alerta a través de los cuales pudo fijarse con más facilidad (Bermúdez-Rattoni y Prado-Alcalá, 2001). En relación a ello, se hallan investigaciones en la literatura que indican que el contenido emocional de los estímulos o eventos influye sobre la memoria de corto y largo plazo tanto en niños como adultos jóvenes o mayores (Cordon, Melinder, Goodman y Edelstein, 2013; Moayeri, Cahill, Jin y Potkin, 2010).

Es necesario tener en cuenta los efectos fisiológicos que la actividad física tiene en el organismo, y como esto puede modular los diferentes procesos de memoria. La actividad aeróbica se relaciona con un aumento en la producción del Factor Neurotrófico Derivado del Cerebro (BDNF, por sus siglas en inglés, Brain Derived Neurotrophic Factor), y con un incremento a nivel noradrenérgico y de los glucocorticoides (Keyan y Bryant 2017a). Cuando se trata de intervenciones agudas de ejercicio se observa una activación del eje hipotalámico-hipofisarioadrenal, elevando los niveles de cortisol, lo cual sería un factor crucial en el fortalecimiento de memorias emocionales. Sin embargo, en estos casos existe una ventana temporal de efectividad, ya que los beneficios se observan únicamente cuando la actividad física es realizada de forma inmediata, ya sea antes o después del aprendizaje (Keyan y Bryant 2017c; Weinberg, Hasni, Shinohara y Duarte, 2014). Por otro lado, y en relación al presente estudio, existen investigaciones que relacionan una vida físicamente activa con el aumento de BDNF en sangre, mejorando las funciones cognitivas y la proliferación de tejido cerebral (Tsai et al., 2014). Adicionalmente, se encontró que aquellos sujetos que poseían los niveles cardiorrespiratorios más elevados (ganancia lograda con el entrenamiento aeróbico cardíaco) también eran quienes poseían los valores plasmáticos basales más altos de BDNF (Tsai, Pan, Chen, Wang y Chou, 2016).

Por todo lo anteriormente expuesto, el objetivo del presente trabajo fue evaluar si este enriquecimiento cerebral, debido a la realización de ejercicio, podría repercutir en la memoria emocional. La mayoría de los estudios emplean diseños donde la actividad deportiva es considerada como un tratamiento o intervención aguda en donde los participantes cumplen diferentes planes aeróbicos y/o de resistencia y se estudia su efecto de modo previo-posterior a la intervención (Keyan y Bryant, 2017a, 2017b, 2017c). Sin embargo, también puede implementarse un diseño en donde se evalúen participantes que posean un estilo de vida comprometido con la actividad física y deportiva, y comparar su desempeño en diversas tareas con el de aquellos participantes que son considerados sedentarios o que dedican poco tiempo de sus vidas a estas prácticas (Albinet, Abou-Dest, André y Audiffren, 2016). El presente estudio se relaciona con la segunda de las líneas de investigación, ya que se dividió a los participantes de acuerdo a su nivel de actividad física y se evaluó su desempeño en una tarea de memoria emocional. En base a los antecedentes acerca de la memoria emocional, la primera de las hipótesis planteadas fue que la información con contenido emocional se recordaría en mayor medida que la neutra. En relación al efecto beneficioso que la actividad física posee sobre las funciones cognitivas, la segunda de las hipótesis predice que los participantes que realizan más actividad física tendrán un mejor desempeño mnemónico que aquellos sujetos considerados sedentarios.

\section{Método}

\section{Participantes}

Inicialmente se contó con la participación voluntaria de 48 adultos, 25 de ellos hombres. Ninguno de los sujetos presentaba enfermedades cardiovasculares crónicas, ni afecciones incapacitantes en cuanto a la realización de actividad física, además no poseían antecedentes psiquiátricos. Los participantes fueron convocados por medio de anuncios en redes sociales y a través de correo electrónico. Cuatro sujetos fueron descartados del análisis final, ya que no pudieron asistir a la instancia diferida de evaluación de memoria. Así, la muestra final estuvo constituida por 44 adultos, 23 de ellos hombres. Estos participantes fueron divididos de acuerdo a su nivel de actividad aeróbica/deportiva en tres grupos (ver 2.2.1 para una descripción de la división de los grupos): Nivel bajo $(n=9$; N1); Nivel moderado $(n=14 ; \mathrm{N} 2)$; Nivel alto $(n=21 ; \mathrm{N} 3)$. En la Tabla 1 se encuentran los datos sociodemográficos de edad, escolarización, peso, $n$ de acuerdo al género y destreza 
Tabla 1

Datos sociodemográficos de los participantes en función de los grupos de actividad física

\begin{tabular}{|c|c|c|c|c|}
\hline & & N1 & N2 & N3 \\
\hline Edad & Rango & $23-47$ & $24-54$ & $18-58$ \\
\hline Edad & Media & $31(D E 8.81)$ & $33.71(D E 9.69)$ & $33.52(D E 11.61)$ \\
\hline Número de & Hombres & 2 & 6 & 15 \\
\hline participantes & Mujeres & 7 & 8 & 6 \\
\hline Escolarización & & $14.78(D E 4.17)$ & $18.69(D E 4.44)$ & $18.29(D E 7.51)$ \\
\hline Peso & & $66.78(D E 5.86)$ & $67.43(D E 4.11)$ & 76.67 (DE 3.55) \\
\hline Número de zurdos & & 0 & 2 & 3 \\
\hline
\end{tabular}

manual. En cuanto a la escolarización los sujetos consignaron la cantidad de años que dedicaron a la educación formal, siendo de modo genérico siete años la cantidad para primario completo, 12 secundario completo, 15 terciario completo, 17/18 o más universitario completo, 18 o más posgrado, y así sucesivamente de acuerdo a la cantidad de años empleados en su educación formal. Adicionalmente se adscriben los datos correspondientes a la destreza manual. No obstante, dicha variable no es relevante a los fines del presente trabajo, ya que la importancia se centra en la realización de actividades y sobre la exigencia percibida por los sujetos que las practican, de tal forma que la eficacia en la ejecución, o la técnica empleada no son consideradas.

Se analizaron cada una de estas medidas y no se hallaron diferencias significativas entre los grupos para ninguna de ellas $(p>.05)$. De modo previo al comienzo del estudio los participantes firmaron un consentimiento informado donde se aseguraba la confidencialidad, así como el anonimato de los datos.

\section{Materiales}

\section{Evaluación del nivel aeróbico}

El Cuestionario Internacional de Actividad Física (IPAQ, por sus siglas en inglés, International Physical Activity Questionnaire) es un instrumento de medición por auto-reporte del tipo y volumen de actividad física realizada, en base a la cantidad de minutos y los días dedicados a ella (IPAQ Research Committee, 2005). Este instrumento se encuentra validado (ver Craig et al., 2003) y se utilizó su versión en español con mínimas modificaciones al castellano rioplatense. La unidad que arroja el IPAQ es el Equivalente Metabólico de Tarea (MET, por sus siglas en inglés, Metabolic Equivalent of Task), este se refiere al consumo energético que representa para un determinado individuo la realización de tareas musculoesqueléticas. Un MET comprende el consumo energético estando sentado serenamente y en posición de descanso. De esta forma, el cuestionario se divide en tres tipos de actividades: vigorosas, cuyo costo es ocho veces superior a permanecer sentado (8 METs); moderadas (costo de 4 METs); caminatas (costo de 3.3 METs). El cuestionario considera la actividad realizada en los últimos siete días y en una semana habitual o rutinaria (considerando los últimos tres meses). En este trabajo, debido a que el objetivo estaba centrado en estilos de vida físicamente activos y deportivos, y no en intervenciones focales, el análisis se basará en los resultados de la semana habitual.

El IPAQ permite obtener valores separados tanto para las dos intensidades de actividad (vigorosa y moderada) como para las caminatas. Estos se registran como METminutos/semana, obtenido del producto entre el valor MET correspondiente (8, 4 ó 3.3 dependiendo de la actividad) * la cantidad de minutos dedicados * la cantidad de días empleados. Ya que el valor multiplicador MET para cada actividad está calculado para personas de 60 kilogramos de peso, se debe introducir un factor adicional cuando el sujeto supere o no alcance esta cantidad. Se obtiene: MET-minutos/semana * (peso en kilogramos/60). Finalmente, los tres valores alcanzados (actividades vigorosas + moderadas + caminatas) se suman para obtener el METminutos/semana total. En base a esto, el IPAQ permite dividir a los sujetos en tres niveles según su gasto energético: bajo, moderado, alto. Estas divisiones surgen 
de información cuantitativa (METs) así como cualitativa (análisis de caso por caso).

Un nivel bajo se alcanza cuando el sujeto no reporta actividades, o bien cuando se reportan actividades, pero no son suficientes para reunir los requisitos de las categorías superiores.

Para formar parte del nivel moderado se deben cumplir algunas de las siguientes condiciones: registrar tres o más días de actividad vigorosa, por al menos 20 minutos cada día; o practicar cinco o más días de actividades moderadas y/o caminatas por al menos 30 minutos cada día; o alcanzar cinco o más días de cualquier combinación de caminatas, actividad moderada o actividad vigorosa acumulando un mínimo de 600 MET-minutos/semana.

El nivel intenso lo constituyen sujetos que registran al menos tres días de actividades vigorosas, alcanzando al menos 1500 MET-minutos/semana; o bien aquellos que alcanzan siete o más días de cualquier combinación de caminatas, actividades moderadas o actividades vigorosas, acumulando al menos 3000 MET-minutos/semana.

\section{Evaluación de la memoria}

La tarea de memoria implicaba que los participantes adquiriesen información visual que luego deberían recordar o reconocer, de modo inmediato y diferido. Por lo que se evaluaban tres variables dependientes: el grado de activación que generaba en los participantes la observación de imágenes con distinta valencia (variable dependiente de Arousal), imágenes recordadas (variable dependiente de recuerdo libre, tanto inmediato como diferido), imágenes reconocidas (variable dependiente de reconocimiento, tanto inmediato como diferido). La información visual consistía en 108 imágenes seleccionadas del Sistema Internacional de Imágenes Afectivas (IAPS, por sus siglas en inglés International Affective Picture System; Lang, Bradley y Cuthbert, 1995). Setenta y dos de esas imágenes eran emocionalmente activantes (36 con valencia positiva y 36 con valencia negativa) y 36 eran imágenes neutras, no activantes. Las mismas fueron seleccionadas de acuerdo a trabajos previos (Cahill, Gorski y Le, 2003; Justel, Psyrdellis y Ruetti, 2014) y cubrían un rango amplio de arousal/activación (de 2.95 a 6.36) y valencia (de 1.97 a 4.93) de acuerdo al manual de Lang et al., (1995). Treinta seis de esas imágenes ( 12 de cada valencia) fueron usadas como imágenes blanco (target) mientras que las otras 72 fueron usadas como imágenes novedosas en las evaluaciones de reconocimiento (inmediato y diferido).

\section{Procedimiento}

El estudio se dividió en dos sesiones, con un intervalo entre ellas de siete días. La sesión uno se encontraba dividida en tres fases consecutivas, la primera de ellas (Informativa) consistió en la firma del consentimiento informado, así como relleno de una planilla de datos sociodemográficos y el IPAQ.

En la siguiente fase (Adquisición) los participantes observaron las 36 imágenes target, una cada siete segundos de modo aleatorio, mientras que lo hacían completaban en una grilla cuan emocional o activante las consideraban, siendo 0 nada emocional/activante y 10 totalmente activante/emocional (variable dependiente de Arousal).

Luego (fase de Evaluación) se les pidió a los sujetos que escribieran cuantas imágenes recordaban de las vistas previamente (variable dependiente de recuerdo libre inmediato), se les dio un lapso de cinco minutos para completar esta tarea. Posteriormente las 36 imágenes originales fueron mezcladas con 36 nuevas de modo aleatorio, de tal forma que los participantes observaban cada estímulo durante cinco segundos, debiendo escribir en una grilla si recordaban haber visto la imagen previamente o no (variable dependiente de reconocimiento inmediato). Todas las tareas de esta primera sesión se llevaron a cabo de forma individual, o en grupos de no más de cuatro personas.

En la segunda sesión (a la semana siguiente de la primera sesión) los participantes volvían a pasar por la fase de evaluación, tanto de recuerdo libre como de reconocimiento (medidas dependientes diferidas). En el reconocimiento se mezclaron las 36 imágenes originales con 36 novedosas diferentes a las usadas en la evaluación de recuerdo libre inmediato. 


\section{Análisis de datos}

\section{Evaluación del nivel aeróbico}

El nivel aeróbico fue evaluado de modo mixto para poder dividir a los participantes en los tres niveles. En primera instancia los datos arrojados por el IPAQ permiten obtener un valor de MET cuantitativo (que multiplica la cantidad de minutos empleados en una actividad, por la cantidad de días y se relativiza de acuerdo al peso del sujeto para las actividades vigorosas, moderadas y caminatas, ver apartado 2.2.1). Este valor MET fue analizado en conjunto con la información cualitativa arrojada por el cuestionario (tipo de actividad y distribución en días $/ \mathrm{mi}$ nutos) para de ese modo indicar el nivel de actividad aeróbica que le correspondía a cada participante. Es decir que para asignar un participante a un nivel se tenían en cuenta los valores MET y la inspección cualitativa de cada cuestionario. Como los datos MET no cumplían con los supuestos de homogeneidad y normalidad se utilizaron análisis no paramétricos para comparar a los tres grupos en cada uno de los tipos de actividad empleando la $U$ de Mann Whitney. Para realizar comparaciones intragrupo se utilizó el test de Wilcoxon.

\section{Evaluación de la memoria}

$\mathrm{El}$ arousal, recuerdo y reconocimiento (inmediato y diferido) fueron analizados de modo independiente mediante un Análisis de Varianza (ANOVA) de medidas repetidas (MR) donde Nivel (1, 2 o 3) fue el factor inter sujeto y las Imágenes (Neutra, Positiva, Negativa) fueron las MR. En este análisis se emplearon evaluaciones paramétricas debido a que los datos cumplían los supuestos de normalidad y homogeneidad.

Se realizaron análisis Post hoc (LSD) para evaluar efectos significativos principales o interacciones. El parcial Eta cuadrado se usó para estimar el tamaño del efecto $\left(\eta^{2} p\right)$. El valor de alfa se situó en .05 y se usó el paquete estadístico SPSS 17.0 para analizar los datos.

\section{Resultados}

\section{Niveles aeróbicos}

En base a las actividades realizadas (divididas en vigorosas, moderadas y caminatas), así como la cantidad de días por semana y la cantidad de horas dedicadas a cada actividad se clasificó a los participantes en cada uno de los

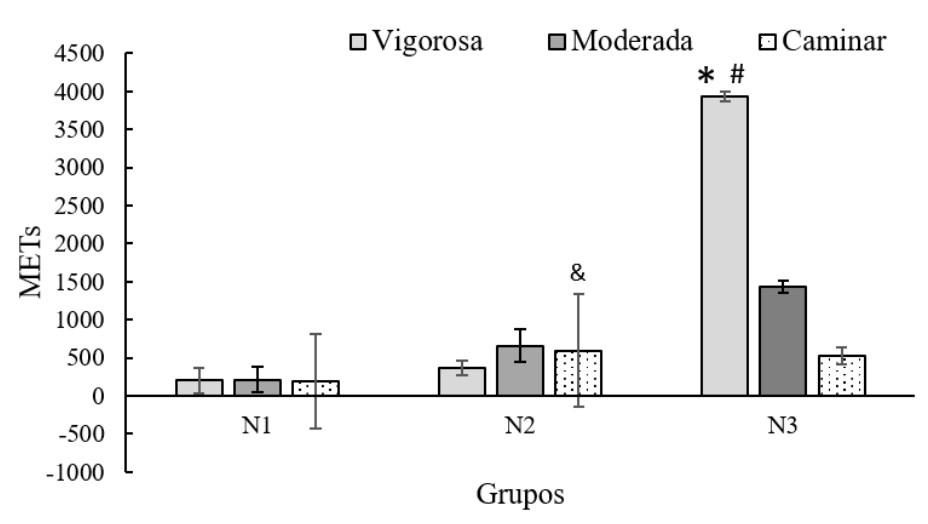

Figura 1. Niveles aeróbicos. Valores de METs para cada tipo de actividad (vigorosa, moderada y caminatas) divididos por cada grupo. N1: Participantes con un nivel bajo de actividad. N2: Sujetos con un nivel moderado de actividad. N3: Participantes con un nivel intenso de actividad. Las líneas verticales representan errores estándar de la media. * Indica diferencias en actividades vigorosas entre N3 vs N1 y N3 vs N2. \# Indica diferencias entre actividades vigorosas vs moderadas y caminata para el grupo N3. \& Indica diferencias en las caminatas entre los grupos N1 y N2. 
niveles. La Figura 1 muestra los niveles de MET para cada tipo de actividad de cada uno de los grupos.

El grupo de Nivel $1(\mathrm{~N} 1)$ o bajo $(n=9)$ tuvo una media total de METs de 601.2 (DE 154.58). En relación a las actividades vigorosas este grupo las realizó en promedio menos de un día a la semana, por un tiempo promedio de 20 minutos (METs = 199.7), la única actividad vigorosa reportada fue futbol. Las actividades moderadas fueron realizadas menos de dos días a la semana, con una media de 20 minutos cada día, con un total de 208.29 METs (ejemplo de estas actividades fue limpiar la casa). En relación a las caminatas, fueron realizadas menos de tres días por semana en promedio, con un tiempo medio de 26 minutos y un total de METs de 193.2.

El grupo de Nivel 2 (N2) o moderado $(n=14)$ tuvo una media de METs de 1582.97 ( $D E$ 208.53). Realizó actividades vigorosas en promedio un día a la semana, por un tiempo medio de 25 minutos, un total de 370.29 METs (en este caso las actividades reportadas fueron: futbol, ir al gimnasio y realizar ciclismo). En relación a las actividades moderadas este grupo las realizó tres veces por semana en promedio, una media de 50 minutos cada día, un total de METs de 658.71 (ejemplos de estas actividades fueron: limpiar la casa, zumba, gimnasia localizada, jugar al ping pong). En relación a las caminatas, este grupo realizó un promedio de cinco días a la semana de esta actividad, con una media de tiempo de 40 minutos, un total de METs de 553.97 .
Mientras que el grupo de Nivel 3 (N3) o alto $(n=21)$ tuvo una media de METs de 5817.44 ( $D E$ 1063.85). Las actividades vigorosas fueron realizadas una media de cuatro días por semana, durante hora y media de promedio, dando un total de 3933.33 METs (ejemplos de actividades en este caso fueron: natación, running, artes marciales, cross fit, entre otras). En relación a las actividades moderadas fueron realizadas por un promedio de cuatro días a la semana, una media de dos horas cada día, dando un total de METs de 1336.03 (ejemplos de estas actividades fueron: limpiar, albañilería, ciclismo). Mientras que las caminatas fueron realizadas una media de tres días por semana, por un promedio de 40 minutos, un total de METs de 519.85 .

En relación a los análisis intergrupales, el grupo N3 tuvo mayor cantidad de METs para actividades vigorosas que el grupo $\mathrm{N} 1, Z=-4.3, p<.0001$, y que el grupo $\mathrm{N} 2$, $Z=-5.01, p<.0001$. Además, se halló una tendencia en relación a las caminatas, siendo realizada en mayor medida por el grupo N3 que N1 $(p=.08)$. El grupo N2 tuvo un nivel mayor de METs para caminatas que el grupo N1 $Z=-2.53, p=.01$.

En relación a los análisis intragrupales, el grupo N3 tuvo mayor cantidad de METs para actividades vigorosas que moderadas $Z=-2.79, p=.005$ y mayor cantidad de METs para actividades vigorosas que caminatas $Z=-4.01$, $p<.0001$. Sin diferencias entre los valores de METs para caminatas y actividades moderadas. No se hallaron diferencias en los METs de los grupos N1 y N2 para cada tipo de actividad realizada por cada grupo, $p>.05$.

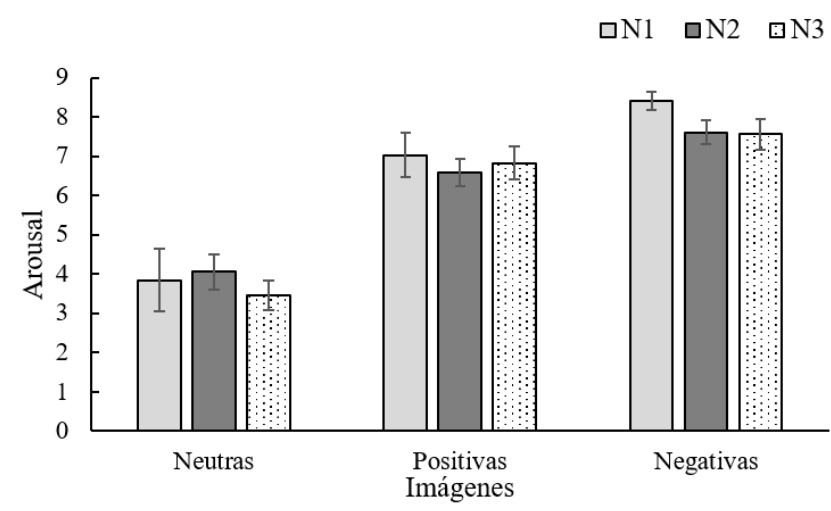

Figura 2. Arousal. Grado de activación que le generaba a los sujetos observar las imágenes neutras, positivas o negativas. N1: Participantes con un nivel bajo de actividad. N2: Sujetos con un nivel moderado de actividad. N3: Participantes con un nivel intenso de actividad. Las líneas verticales representan errores estándar de la media. 


\section{Arousal}

La primera variable dependiente analizada fue el nivel de arousal o activación que les producían las imágenes observadas a los sujetos. El ANOVA arrojó un efecto significativo de Imágenes $F(2,82)=107.69, p<.0001$, $\eta^{2} \mathrm{p}=.724$. Los análisis Post hoc indicaron un nivel de activación ascendente siendo las neutras las imágenes menos activantes, seguidas por las positivas y por último las negativas. Ningún otro análisis indicó diferencias estadísticamente significativas, $p>.05$. Los resultados se encuentran representados en la Figura 2.

\section{Recuerdo libre inmediato}

Luego de observar y calificar las imágenes, los participantes tenían que escribir aquellas que recordaban haber visto. El ANOVA indicó un efecto significativo de Imágenes $F(2,82)=24.23, p<.0001, \eta_{\mathrm{p}}^{2}=.371$, donde las imágenes negativas fueron mayormente recordadas que las positivas y éstas últimas a su vez mayormente recordadas que las neutras. Además se halló que la interacción de Imágenes $\mathrm{x}$ Nivel también resultó significativa $F(2$, $84)=2.59, p=.042, \eta^{2} \mathrm{p}=.112$. Los análisis Post hoc in- dicaron que los participantes del Nivel 3 recordaron mayor cantidad de imágenes positivas que los sujetos del Nivel $1, p=.019$ y del Nivel $2, p=.010$. Resumiendo, las imágenes emocionales se recordaron más que las neutras y esto a su vez se vio afectado por el nivel de actividad deportiva que el sujeto poseía, dando cuenta que aquellos participantes con un mayor nivel de entrenamiento tuvieron un mejor recuerdo de información con contenido positivo (Figura 3).

\section{Reconocimiento inmediato}

Luego los sujetos debían indicar, dentro un grupo de 72 imágenes, cuáles reconocían haber visto entre aquellas novedosas. Los falsos reconocimientos se sustrajeron del reconocimiento total (para cada tipo de imagen). Los resultados se encuentran en la Tabla 2. El ANOVA indicó un efecto significativo de Imágenes $F(2,82)=3.22$, $p=.045, \eta_{\mathrm{p}}^{2}=.073$, donde las imágenes neutras fueron mayormente reconocidas que las emocionales. Además se halló un efecto significativo de Nivel $F(2,41)=4.13$, $p=.023, \eta_{\mathrm{p}}^{2}=.168$, los análisis Post hoc indicaron que el grupo de Nivel 3 reconoció mayor cantidad de imágenes que el grupo del Nivel $1, p=.043$ y que el del Nivel 2, $p=.014$.

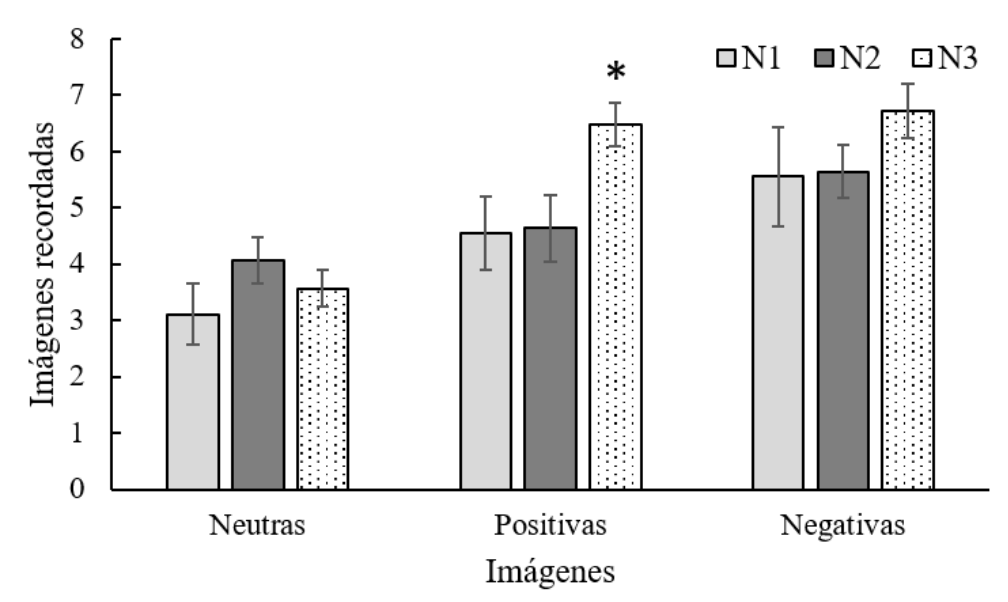

Figura 3. Recuerdo Libre Inmediato. Media de Imágenes neutras, positivas o negativas recordadas por los distintos grupos. N1: Participantes con un nivel bajo de actividad. N2: Sujetos con un nivel moderado de actividad. N3: Participantes con un nivel intenso de actividad. Las líneas verticales representan errores estándar de la media. * Indica diferencias significativas entre el grupo N3 vs N1 y N3 vs N2 para las imágenes positivas. 
Tabla 2

Reconocimiento inmediato de imágenes en cada uno de los grupos

\begin{tabular}{cccc}
\hline Grupos & Neutras & Positivas & Negativas \\
\hline N1 & $11.22(0.46)$ & $11.33(0.33)$ & $10.78(0.43)$ \\
N2 & $11.57(0.23)$ & $10.71(0.30)$ & $10.93(0.49)$ \\
N3 & $11.90(0.07)$ & $11.55(0.19)$ & $11.90(0.07)$ \\
\hline
\end{tabular}

Nota. Medias de imágenes reconocidas (desvíos estándar entre paréntesis). N1: Participantes con un nivel bajo de actividad. N2: Sujetos con un nivel moderado de actividad. N3: Participantes con un nivel intenso de actividad.

\section{Reconocimiento libre diferido}

Siete días después de la primera sesión volvió a evaluarse la memoria, para lo cual los participantes debían anotar que imágenes recordaban de las vistas la semana anterior. El ANOVA indicó un efecto significativo de Imágenes $F(2,80)=16.56, p<.0001, \eta_{\mathrm{p}}^{2}=.293$; las imágenes negativas se recordaron más que las positivas y éstas a su vez más que las neutras (Figura 4). Ningún otro análisis arrojó diferencias estadísticamente significativas, $p>.05$.

\section{Reconocimiento diferido}

Posteriormente a la fase de recuerdo libre, los participantes debían diferenciar de un conjunto de 72 imágenes aquellas que habían observado la semana anterior, de aquellas novedosas. Los análisis estadísticos no arrojaron diferencias estadísticamente significativas en esta medida $p>.05$. Los resultados se encuentran en la Tabla 3.

Tabla 3

Reconocimiento diferido de imágenes en los tres grupos

\begin{tabular}{cccc}
\hline Grupos & Neutras & Positivas & Negativas \\
\hline N1 & $11.89(0.77)$ & $11.00(0.37)$ & $10.78(0.46)$ \\
N2 & $11.43(0.18)$ & $11.38(0.18)$ & $10.67(0.26)$ \\
N3 & $10.64(0.62)$ & $11.36(0.40)$ & $10.36(0.38)$ \\
\hline
\end{tabular}

Nota: Medias de imágenes reconocidas (desvíos estándar entre paréntesis). N1: Participantes con un nivel bajo de actividad. N2: Sujetos con un nivel moderado de actividad. N3: Participantes con un nivel Intenso de actividad.

\section{Discusión}

La actividad aeróbica deportiva es un área de creciente interés para la investigación, ya que podría implementarse como estrategia para mejorar las funciones cognitivas (Barha et al., 2017; Erickson et al., 2011), por ello el ob-

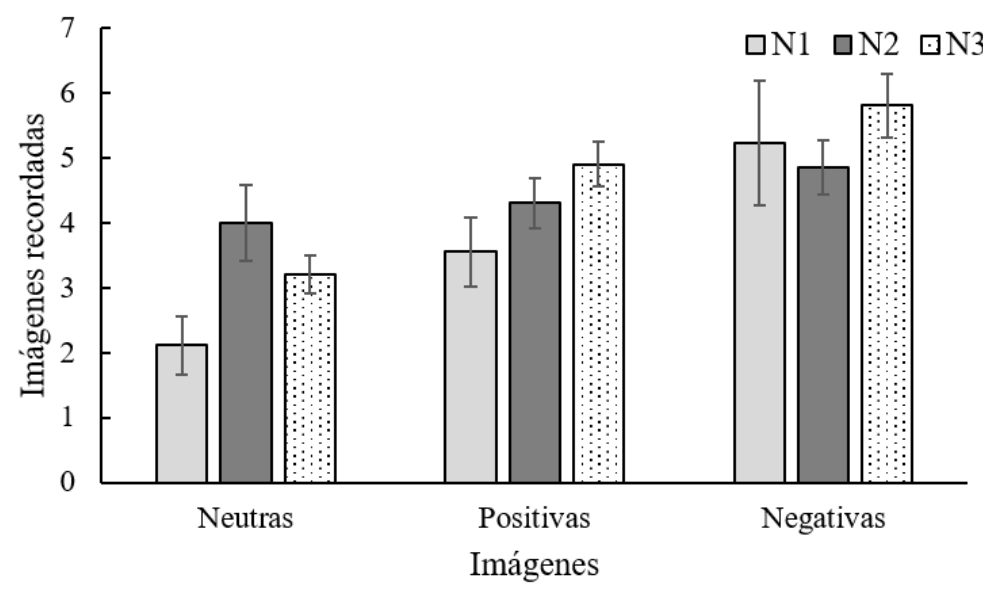

Figura 4. Recuerdo Libre Diferido. Media de Imágenes neutras, positivas o negativas recordadas por los distintos grupos. N1: Participantes con un nivel bajo de actividad. N2: Sujetos con un nivel moderado de actividad. N3: Participantes con un nivel intenso de actividad. Las líneas verticales representan errores estándar de la media. 
jetivo de este trabajo fue evaluar el efecto de una vida deportiva activa sobre la memoria emocional. En primer lugar, se halló que la información emocional se recordó en mayor medida que la neutra y en segundo lugar, se encontró que este patrón de resultados se vio afectado por el nivel aeróbico/físico que poseía el participante. Ambos resultados apoyarían las hipótesis planteadas. A continuación se discutirán en mayor detalle estos hallazgos.

La primera de las hipótesis planteadas fue que la información con contenido emocional sería mayormente recordada que la neutra. Efectivamente, las imágenes emocionales fueron calificadas como más activantes que las neutras y este nivel de emocionalidad/activación repercutió en el recuerdo, ya que las imágenes emocionales fueron mayormente recordadas que las neutras, tanto de modo inmediato como diferido (Hämmerer et al., 2017; Justel et al., 2014; Schümann, Bayer, Talmi y Sommer, 2018). Hay explicaciones posibles a porqué la información emocional es recordada en mayor medida al ser comparada con información neutra, por ejemplo, se les presta mayor atención a los detalles (McGaugh y Roozendaal, 2009). A nivel fisiológico, durante las situaciones emocionales se liberan hormonas adrenales, tales como la adrenalina, la noradrenalina y los glucocorticoides. La acción de estas hormonas es una de las claves para llegar a la comprensión de las relaciones entre la memoria y las emociones, ya que las mismas modulan selectivamente la memoria de eventos que son emocionalmente significativos (McGaugh y Roozendaal, 2002; Roozendaal, 2000; Taylor, Ellenbogen, Washburn y Joober, 2011).

La segunda de las hipótesis planteadas indicaba que el nivel de actividad deportiva de un sujeto influiría sobre su desempeño mnemónico. Esta hipótesis se sostuvo de modo parcial ya que se halló que tanto el recuerdo libre como el reconocimiento inmediato se vieron afectados por el nivel deportivo de los participantes, pero no así el recuerdo y reconocimiento diferido. Los sujetos que tenían un nivel de actividad intenso, implicando una actividad física y deportiva demandante en su vida diaria, tuvieron un mejor recuerdo libre inmediato que los sujetos con un nivel bajo o moderado. Más aun, este mejor recuerdo fue sólo para la información con valencia positiva, no así la neutra o negativa.

En relación al reconocimiento, fue un resultado que no esperábamos, visto y considerando que eran adultos de edad temprana a media y por lo general el reconocimiento en este tipo de población suele tener un efecto de techo. Sin embargo, los resultados indicaron que los participantes con un nivel de actividad intensa tuvieron un mejor desempeño en esta tarea de memoria que los otros dos grupos de sujetos.

El motivo por el cual no se obtuvieron resultados en la medida diferida necesita de mayores estudios para poder dar una respuesta precisa. Sin embargo, podría hipotetizarse, y señalar como posible limitación del presente trabajo, que el $n$ empleado es pequeño así como dispar en los grupos estudiados. Además, es de resaltar como otra posible limitación que no se encontraba emparejada la cantidad de mujeres y hombres en cada uno de los niveles, sin embargo cabe destacar que no se hallaron diferencias significativas en esta medida.

Por otro lado, la literatura indica que habría un efecto de dosis-respuesta, por tanto, se observaría un mejor desempeño cognitivo cuanto más ejercicio se realiza (Masley, Roetzheim y Gualtieri, 2009). En nuestros resultados encontramos que hay una diferencia entre los niveles bajo y moderado por un lado e intenso por el otro. Sin embargo, no encontramos diferencias entre los dos primeros niveles. Una posible explicación, así como limitación del presente estudio, es que las condiciones baja y moderada son muy similares entre sí diferenciándose de la condición intensa. En cuestión de METs la baja tiene un total de 601, la moderada de 1582 mientras que la intensa de 5817. Sería interesante contar con una muestra de participantes que estuviera en un nivel con valores intermedios entre las categorías moderada e intensa manejadas en el presente trabajo, o un nivel bajo que posea menos de 300 MET y estudiar si en esos casos se hallaría un desempeño dosis-respuesta de actividad deportiva.

Otra posible limitación del presente trabajo se relaciona con el cuestionario, que si bien posee una delimitación clara en cuanto a las actividades para cada nivel es en última instancia el sujeto (mediante su auto reporte) quién 
determina si la actividad a la que refiere pertenece a una $u$ otra categoría. Esto podría generar inconvenientes a la hora de calcular los valores METs que corresponden a cada participante. Sin embargo, esta contrariedad podría subsanarse utilizando sujetos expertos en su disciplina, y realizando una comparación entre sus desempeños y los observados en sujetos sedentarios, lo cual plantearía un futuro estudio posible. También sería interesante poder evaluar diferentes grupos dentro de cada nivel; por ejemplo, participantes con un entrenamiento intenso en deportes de grupo vs participantes con un nivel intenso en deportes de resistencia o trabajo en solitario. Los datos al respecto indican que los primeros, denominados deportistas de habilidades abiertas, desarrollan niveles altos de control ejecutivo y utilizan mejor la información que les brinda el ambiente. Como resultado, obtienen tiempos de respuesta más cortos al compararlos con deportistas de habilidades cerradas. Tal efecto puede deberse al hecho de que estos últimos se desenvuelven en ambientes que por lo general se mantienen estables. Esto los lleva a mantener movimientos esquemáticos y repetitivos durante prácticamente toda la actividad. En comparación, los deportes en equipo presentan ambientes en constante cambio, que requieren cambios de estrategias y adaptaciones sucesivas por parte de los jugadores (Yu, Chan, Chau y Fu, 2017).

Otra posible comparación, así como futuro estudio, sería entre actividades que implican un compromiso cognitivo o no, ya que los datos de la literatura indican que cuanto mayor el compromiso cognitivo implicado en el deporte realizado, mejor el rendimiento en las pruebas evaluadas (Jäger et al., 2015), es decir que no sólo es relevante el realizar actividad sino también el tipo de actividad que se realice.

Como se mencionó en la introducción, se ha encontrado que el hipocampo se encuentra más desarrollado en aquellas personas que tienen un mejor nivel cardiorrespiratorio fruto del entrenamiento aeróbico (Erickson et al., 2009; Honea et al., 2009). Además, en la adultez mayor esta estructura se reduce llevando a un deterioro en la memoria y riesgo de demencia; sin embargo, si los adultos mayores siguen un entrenamiento aeróbico se halló que se atenúa este declinamiento cognitivo (Erickson et al., 2009), dando cuenta de la relevancia de mantener un estilo de vida activo a lo largo de la vida. Se estima que para
2050 habrá 114 millones de personas con demencia, siendo una de las mayores causas de discapacidad y dependencia de la población de adultos mayores (Iuliano et al., 2016). Por ende, proponer intervenciones que mejoren nuestra reserva cognitiva es un tópico de capital interés para la salud biopsicosocial de nuestra especie. Promoviendo desde la infancia temprana hasta la adultez mayor, en cada una de las etapas, aquellos tratamientos que pudieran mejorar nuestras capacidades cognitivas, previniendo o retrasando los déficits cognitivos. En relación a ello, la evidencia actual sugiere que realizar ejercicio físico es una intervención prometedora para reducir el deterioro cognitivo (Fallah et al., 2013; Middleton, Barnes, Lui y Yaffe, 2010; Ratey y Loehr, 2011); las personas que se mantienen activas muestran un menor declive de sus funciones cognitivas cuando son mayores (Masley et al., 2009). Además, realizar actividad física es una intervención atrayente debido a que puede ser fácilmente realizable por prácticamente cualquier tipo de población. 


\section{Referencias}

Albinet, C. T., Abou-Dest, A., André, N. y Audiffren, M. (2016). Executive Functions Improvement Following a 5-month Aquaerobics Program in Older Adults: Role of Cardiac Vagal Control in Inhibition Performance. Biological Psychology, 115, 69-77. https://doi.org/10.1016/j.biopsycho.2016.01.010

Barha, C., Falck, R., Davis, J., Nagamatsu, L. y LiuAmbrose, T. (2017). Sex Differences in Aerobic Exercise Efficacy to Improve Cognition: A Systematic Review and Meta-Analysis of Studies in Older Rodents. Frontiers in Neuroendocrinology, 46, 86-105. https://doi.org/10.1016/j.yfrne.2017.06.001

Bermúdez-Rattoni, F. y Prado-Alcalá, R. (2001). Memoria. ¿En dónde está y cómo se forma? [Where is it and How is it Formed?] México: Trillas.

Cahill, L., Gorski, L. y Le, K. (2003). Stress: Interaction with the Degree of Arousal at Encoding Enhanced Human Memory Consolidation with Post-Learning. Learning y Memory, 10, 270-274. https://doi.org/10.1101/lm.62403

Chang, Y. K., Chi, L., Etnier, J. L., Wang, C. C., Chu, C. H. y Zhou, C. (2014). Effect of Acute Aerobic Exercise on Cognitive Performance: Role of Cardiovascular Fitness. Psychology of Sport and Exercise, 15(5), 464-470. https://doi.org/10.1016/j.psychsport.2014.04.007

Chen, A. G., Yan, J., Yin, H. C., Pan, C. Y. y Chang Y. K. (2014). Effects of Acute Aerobic Exercise on Multiple Aspects of Executive Function in Preadolescent Children. Psychology of Sport and Exercise, 15(6), 627-636. https://doi.org/10.1016/j.psychsport.2014.06.004

Colcombe, S. J., Erickson, K., Scalf, P. E., Kim, J. S., Prakash, R., McAuley, E., ... Kramer, A. F. (2006). Aerobic Exercise Training Increases Brain Volume in Aging Humans. The Journals of
Gerontology. Series A, Biological Sciences and Medical Sciences, 61, 1166-1170.

Cordon, I., Melinder, A., Goodman, G. y Edelstein, R. (2013). Children's and Adults' Memory for Emotional Pictures: Examining Age-Related Patterns Using the Developmental Affective Photo System. Journal of Experimental Child Psychology, 114(2), 339-356. https://doi.org/10.1016/j.jecp.2012.08.004

Craig, C. L., Marshall, A. L., Sjöström, M., Bauman, A. E., Booth, M. L., Ainsworth, B. E., ... Oja, P. (2003). International Physical Activity Questionnaire: 12Country Reliability and Validity. Medicine and Science in Sports and Exercise, 35(8), 1381-1395. https://doi.org/10.1249/01.MSS.0000078924.61453. FB

Erickson, K., Prakash, R. S., Voss, M. W., Chaddock, L., Hu, L., Morris, K. S., ... Kramer, A. F. (2009). Aerobic Fitness is Associated with Hippocampal Volume in Elderly Humans. Hippocampus, 19, 1030- https://doi.org/1039. 10.1002/hipo.20547

Erickson, K., Voss, M., Prakash, R., Basak, C., Szabo, A., Chaddock, L. Kramer, A. (2011). Exercise Training increases Size of Hippocampus and Improves memory. Proceedings of the National academy of Sciences of the United States of America, 108(7), 3017-3022.

https://doi.org/10.1073/pnas.1015950108

Fallah, N., Hsu, C., Bolandzadeh, N., Davis, J., Beattie, L., Graf, P. y Liu-Ambrose, T. (2013). A Multistate Model of Cognitive Dynamics in Relation to Resistance Training: The Contribution of Baseline Function. Annals of Epidemiology, 23, 463-468. https://doi.org/10.1016/j.annepidem.2013.05.008

Hämmerer, D., Hopkins, A., Betts, M. J., Maaß, A., Dolan, R. J. y Düzel, E. (2017). Emotional Arousal and Recognition Memory are Differentially Reflected in Pupil Diameter Responses during Emotional Memory for Negative Events in Younger and Older Adults. Neurobiology of Aging, 58, 129-139. https://doi.org/10.1016/j.neurobiolaging.2017.06.02 
Hayes, S. M., Forman, D. E. y Verfaellie, M. (2014). Cardiorespiratory Fitness is Associated with Cognitive Performance in Older but not Younger Adults. The Journals of Gerontology Series B: Psychological Sciences and Social Sciences, 71(3), 474-482. https://doi.org/10.1093/geronb/gbu167

Hogan, C. L., Mata, J. y Carstensen L. L. (2013). Exercise Holds Immediate Benefits for Affect and Cognition in Younger and Older Adults. Psychology and Aging, 28(2), 587-594. https://doi.org/10.1037/a0032634

Honea R. A., Thomas, G. P., Harsha, A., Anderson, H. S., Donnelly, J. E., Brooks, W. M. y Burns, J. M. (2009). Cardiorespiratory Fitness and Preserved Medial Temporal Lobe Volume in Alzheimer's Disease. Alzheimer Disease and Associated Disorders, 23,188-197. https://doi.org/10.1097/WAD.0b013e31819cb8a2

Hwang, J., Brothers, R. M., Castelli, D. M., Glowacki, E. M., Chen, Y. T., Salinas, M., ... Calvert, H. G. (2016). Acute High-Intensity Exercise-Induced Cognitive Enhancement and Brain-Derived Neurotrophic Factor in young, Healthy Adults. Neuroscience Letters, 630, 247-253. https://doi.org/10.1016/j.neulet.2016.07.033

IPAQ Research Committee (2005). International Physical Activity Questionnaire (IPAQ) scoring protocol. Recuperado de http:// www.ipaq.ki.se/scoring.pdf (accessed 01.05.13).

Iuliano, E., di Cagno, A., Aquino, G., Fiorilli, G., Mignogna, P., Calcagno, G. y Di Costanzo, A. (2016). Effects of Different Types of Physical Activity on the Cognitive Functions and Attention in Older People: A Randomized Controlled Study. Experimental Gerontology. https://doi.org/10.1016/j.exger.2015.07.008

Jäger, K., Schmidt, M., Conzelmann, A. y Roebers, C. M. (2015). The effects of Qualitatively Different Acute Physical Activity Interventions in Real-World Settings on Executive Functions in Preadolescent
Children. Mental Health and Physical Activity, 9, 19. https://doi.org/10.1016/j.mhpa.2015.05.002

Justel, N., Psyrdellis, M. y Ruetti, E. (2014). Evaluación y modulación de la memoria emocional: Un estudio preliminar [Evaluation and Modulation of Emotional Memory: A Preliminary Study]. Anuario de Investigaciones de la Facultad de Psicología, XX, 365-368.

Keyan, D. y Bryant, R. A. (2017a). Acute Physical exercise in Humans Enhances Reconsolidation of Emotional Memories. Psychoneuroendocrinology, 77 , $150-157$ https://doi.org/10.1016/j.psyneuen.2017.09.019.

Keyan, D. y Bryant, R. A. (2017b). Brief Exercise Enhances Intrusive Memories of Traumatic Stimuli. Neurobiology of Learning and Memory, 141, 9-13. https://doi.org/.1016/j.nlm.2017.03.012.

Keyan, D. y Bryant, R. A. (2017c). Role of BDNF val66met polymorphism in modulating exercisedinduced emotional memories. Psychoneuroendocrinology, $\quad 77, \quad 150-157$. https://doi.org/10.1016/j.psyneuen.2016.12.013.

Kimura, K. y Hozumi, N. (2012). Investigating the acute effect of an aerobic dance exercise program on neuro-cognitive function in the elderly. Psychology of Sport and Exercise, 13(5), 623-629. https://doi.org/ 10.1016/j.psychsport.2012.04.001

Labelle, V., Bosquet, L., Mekary, S. y Bherer, L. (2013). Decline in executive control during acute bouts of exercise as a function of exercise intensity and fitness level. Brain and Cognition, 81(1), 10-17. https://doi.org/10.1016/j.bandc.2012.10.001

Lang, P. J., Bradley, M. M. y Cuthbert, B. N. (1995). International affective picture system (IAPS): affective ratings of pictures and instruction manual. Technical Report A-6. Gainesville, FL: University of Florida. 
Lee, T. M. C., Wong, M. L., Lau, B. W. M., Lee, J. C. D., Yau, S. Y. y So, K. F. (2014). Aerobic exercise interacts with neurotrophic factors to predict cognitive functioning in adolescents. Psychoneuroendocrinology, 39(1), 214-224. https://doi.org/10.1016/j.psyneuen.2013.09.019

Masley, S., Roetzheim, R. y Gualtieri, T. (2009). Aerobic exercise enhances cognitive flexibility. Journal of Clinical Psychology and Medical Settings, 16, 186193. https://doi.org/10.1007/s10880-009-9159-6

McGaugh, J.L. y Roozendaal, B. (2002). Role of adrenal stress hormones in forming lasting memories in the brain. Current Opinion in Neurobiology, 12(2), 205210. https://doi.org/10.1016/S0959-4388(02)003069

McGaugh, J.L. y Roozendaal, B. (2009). Emotional hormones and memory modulation. Encyclopedia of neuroscience,

933-940. https://doi.org/10.1016/B978-008045046-9.00849-4

Middleton, L. E., Barnes, D. E., Lui, L. Y. y Yaffe, K. (2010). Physical activity over the life course and its association with cognitive performance and impairment in old age. Journal of the American Geriatrics Society, 58(7), 1322e6. https://doi.org/10.1111/j.1532-5415.2010.02903.x

Moayeri, S., Cahill, 1., Jin, I. y Potkin, S. (2010). Relative sparing of emotionally influenced memory in Alzheimer's disease. Neuroreport, 11(4), 653-655. https://doi.org/10.1097/00001756-20000320000001

Ratey J. J. y Loehr J. E. (2011). The positive impact of physical activity on cognition during adulthood: a review of underlying mechanisms, evidence and recommendations. Reviews of Neuroscience, 22(2), 171e85. https://doi.org/10.1515/RNS.2011.017

Roozendaal, B. (2000). Glucocorticoids and the regulation of memory consolidation. Psychoneuroendocrinology, 25(3), 213-238. doi: 10.1016/S0306-4530(99)00058-X
Ruetti, E., Justel, N. y Bentosela, M. (2009). Perspectivas clásicas y contemporáneas acerca de la memoria [Classic and contemporary perspectives on memory]. Suma Psicológica, 16(1), 65-83.

Schümann, D., Bayer, J., Talmi, D. y Sommer, T. (2018). Dissociation of immediate and delayed effects of emotional on episodic memory. Neurobiology of Learning and Memory, 148, 11-19. https://doi.org/10.1016/j.nlm.2017.12.007

Soga, K., Shishido, T. y Nagatomi, R. (2015). Executive function during and after acute moderate aerobic exercise in adolescents. Psychology of Sport and Exercise, 16(P3), 7-17. https://doi.org/10.1016/j.psychsport.2014.08.010

Squire, L. R. (1987). Memory and Brain. New York: Oxford University Press.

Taylor, V., Ellenbogen, M., Washburn, D. y Joober, R. (2011). The effects of glucocorticoids on the inhibition of emotional information: A doseresponse study. Biological Psychology, 86(1), 17-25. https://doi.org/10.1016/j.biopsycho.2010.10.001

Tsai, C. L., Chen, F. C., Pan, C. Y., Wang, C. H., Huang, T. H. y Chen, T. C. (2014). Impact of acute aerobic exercise and cardiorespiratory fitness on visuospatial attention performance and serum BDNF levels. Psychoneuroendocrinology, 45, 121-131. https://doi.org/10.1016/j.psyneuen.2013.12.014

Tsai, C. L., Pan, C. Y., Chen, F. C., Wang, C. H. y Chou, F. Y. (2016). Effects of acute aerobic exercise on a task-switching protocol and brain-derived neurotrophic factor concentrations in young adults with different levels of cardiorespiratory fitness. Experimental Physiology, $\quad 101(7), \quad 836-850$. https://doi.org/10.1113/EP085682

Tulving, E. (2002). Episodic memory: from mind to brain. Annual Review of Psychology, 53, 1-25.

Van Praag, H., Shubert, T., Zhao, C. y Gage, F. H. (2005). Exercise enhances learning and hippocampal 
neurogenesis in aged mice. Journal of Neuroscience, 25, 8680-8685. https://doi.org/10.1523/JNEUROSCI.1731-05.2005 with selective attention in healthy male high-school students. Frontiers in Human Neuroscience, 11, 330. https://doi.org/10.3389/fnhum.2017.00330
Weinberg, L., Hasni, A., Shinohara, M. y Duarte, A. (2014). A single bout of resistance exercise can enhance episodic memory performance. Acta Psychologica, 133, 13. https://doi.org/10.1016/j.actpsy.2014.06.011

Wengaard, E., Kristoffersen, M., Harris, A. y Gundersen, H. (2017). Cardiorespiratory fitness is associated
Yu, Q., Chan, C. C. H., Chau, B. y Fu, A. S. N. (2017). Motor skill experience modulates executive control for task switching. Acta Psychologica, 180, 88-97. https://doi.org/10.1016/j.actpsy.2017.08.013x

\title{
IMPACT OF AN ACTIVE LIFE-STYLE ON EMOTIONAL MEMORY
}

\author{
MAXIMILIANO BOSSIO ${ }^{1}$ Y NADIA JUSTEL ${ }^{2}$
}

\section{EXTENDED SUMMARY}

\section{Introduction}

Over the last years physical activity has driven the attention of researchers. For a long period of time, physically active practices have been linked with benefits regarding the people's global health. Moreover, nowadays it was found that it would modulate cognitive functions both in humans and animals. It has been discovered that acute doses of physical activity, understood as an exposition that does not exceed the hour of work and it happens in a unique moment and at a specific time, as well as the fact of having done sports throughout life, has beneficial effects on cognitive functions of several populations. Additionally, it has been found that the hippocampus and temporal regions are bigger in subjects that have a more developed cardiorespiratory system, because of their aerobic training, than sedentary people. Those structures are relevant for the formation and consolidation of memories, and in relation to this it was found that physical exercise im- proves learning and memory, enhances cellular proliferation and survival of the hippocampus, and also increases white and grey matter. Regarding memory, over the years multiple classifications about memory systems has been proposed, yet none of them could be considered totally satisfying. Despite that, one of the most accepted classifications is the one that divides between explicit and implicit memory. Emotional memory could be considered as a subdivision of explicit memory, and it is the result of the storage of information that was accompanied with stress or alarm factors, through which the memory could fixed more easily. Simultaneously, it is necessary to take into account the physiological effects of physical activity in the organism and how those effects could modulate the different memory processes. Physical activity is related to the Brain Derived Neurotrophic Factor (BDNF) production, and it is also related to an increase on the noradrenergic and glucocorticoid levels.

With acute exercise interventions it is observed an activation of the hypothalamus-pituitary-adrenal axis, which increases cortisol levels, which in turn are a key factor for the enhancement of emotional memories. On the other 
hand, and related with this study, it was found that those subjects that had a more elevated cardiorespiratory levels (profit achieved with the cardiac aerobic training) were also the subjects that had the more elevated BDNF levels, which in turn enhance cognitive functions and increase brain tissue proliferation. Based on this background, the aim of this study was to evaluate if this brain enrichment, as a consequence of physical activity performance, could modulate emotional memory. Related to the literature about emotional memory, the first hypothesis was that information with emotional content would be better remembered than the neutral one. Concerning the beneficial effects that physical activity has on cognitive functions; the second hypothesis predicts that participants who do more physical activity would have a better mnemonic performance than those subjects who were sedentary.

\section{Methodology}

Forty-four adult subjects participated of this study, 23 male. None of them had chronic cardiovascular diseases, nor disabling conditions regarding physical activity, besides they did not have psychiatric antecedents. All of them were recruited through social media advertisement and through e-mail. Participants were divided according to their physical activity level into three groups: Low level $(n=9 ; \mathrm{N} 1)$; Moderate level $(n=14 ; \mathrm{N} 2)$; and High level $(n=21 ;$ N3). Previous to the start of the study, the participants signed an informed consent where the confidentiality of the data was assured as well as its anonymity. The sociodemographic information, including age, educational level, weight, $n$ for gender, and handedness, was analyzed, without finding significant differences in any of these measures. Concerning the evaluation of the aerobic levels, the International Physical Activity Questionnaire (IPAQ) was employed, which enables to obtain a quantity unit defined as the Metabolic Equivalent of Task (MET). The METs characterize the energy-consumption that represents the performance of musculoskeletal tasks for an individual. A MET includes the energy-consumption while being seated quietly and in resting position. Thereby, the questionnaire is divided in three types of activities: vigorous, its cost is 8 times superior to stay seated ( 8 METs); moderate (cost of 4 METs); walks (cost of 3.3 METs). Because this work was focus on physically active ways of living, an analysis based on the activities that participants carried out in a regular or routine week of their lives (considering the last 3 months) was performed. The IPAQ enabled us to divide the participants in three levels according to their energy-consumption: low, moderate and high. These divisions arise from quantitative information (METs) and also qualitative information (analysis of each participant). Additionally, the memory task implied that the participants acquired visual information which then they had to recall and recognize, both immediately and deferred. Thus, three dependent variables were evaluated: arousal level that the observation of the images produced in the participants (dependent variable of Arousal), images recalled (dependent variable of Free Recall, immediate and deferred), and images recognized (dependent variable of Recognition, immediate and deferred). The visual information consisted of 108 images selected from the International Affective Picture System (IAPS). Seventy-two of those images were emotionally activating (36 with positive valence and 36 with negative valence) and 36 were neutral, non-activating images. Consequently, the study was divided in two sessions, with a 7-day interval between them. The first session was divided in three consecutive phases, the first of them (Information) consisted in the signed of the informed consent, the filling-out of the sociodemographic questionnaire and the IPAQ. This phase was followed by the Acquisition Phase, where the participants watched the 36 target images, while they completed a spreadsheet where they indicated how emotional or activating the images were for them, being 0 non-emotional/activating and 10 completely emotional/activating (dependent variable of arousal). Then, during the Evaluation phase, they were requested to write the images they had previously seen (dependent variable of immediate recall). Later, the 36 target images were mixed with 36 new ones in a random order, and the participants had to write whether they recognized seeing those images or not (dependent variable of immediate recognition). In the second session (a week after the first session) the participants had another evaluation phase, where recall and recognition were evaluated again (deferred measures). In this deferred recognition task, the 36 target images were mixed with 36 new ones, which were different to the ones used in the immediate recognition task. 


\section{Results}

Based on the activities performed (divided in vigorous, moderate and walks), the amount of days per week, and the amount of hours dedicated to each activity the participants were classified in their respectively levels: the level 1 group (N1) or low level $(n=9)$ had a MET media of 601.2 (SD 154.58); the level 2 group (N2) or moderate level $(n=14)$ had a MET media of 1582.97 (SD 208.53); while the level 3 group (N3) or high $(n=21)$ had a MET media of 5817.44 ( $S D$ 1063.85). Regarding the intergroup analysis, the N3 group had a larger amount of METs for vigorous activities than the N1 and N2 group. Besides, the N2 group had a greater level of METs for walks than the N1 group. Related to the intra-group analysis the N3 group had a larger amount of METs for vigorous activities than for moderate, and a greater amount of METs for vigorous activities than walks. On the other hand, related to Arousal, it was observed an ascendant level of activation, being the neutral images the least activating images, followed by the positive ones and the negatives as the most activating pictures. As to immediate free recall refers, the emotional images were better remembered than the neutral ones, and this pattern of recall was affected by the degree of physical activity that the subject had, indicating that those participants with a high level of training had a better recall of positive information than the other participants. Then, the participants had to indicate from a pool of 72 images which ones they recognized as previously seen. False recognition were subtracted from the total recognition for each type of image (positive, negative and neutral). The results indicated that the neutral images were better recognized than the emotional ones and this pattern of recognition was affected by the physical level of the subject, being the level 3 group the one that had the better recognition of the three groups.

\section{Discussion}

Aerobic activity is an area of growing interest for research, since it could be implemented as a strategy to improve cognitive functions, because of that the goal of this work was to evaluate the effect that an aerobic, active life had on emotional memory. The first of the hypothesis raised was that the information with emotional content would be better remembered than the neutral one. In fact, the emotional images were rated as more activating than the neutral ones, and this level of emotionality affected the recall, since the emotional pictures were better remembered than the neutral ones, both immediately and deferred. The second hypothesis raised that the physical level of the subjects would affect their mnemonic performance. This hypothesis was only partially supported since the immediate recall and recognition were affected by the physical level of the participants, but not the deferred measures. The subjects that had an intense physical level, which implicated a physical and demanding aerobic life, had a better immediate recall than the participants with a low or moderate level. Furthermore, this better recall was only found for the positive information, not the neutral or negative. The reason why no results were obtained in the deferred measure needs further research to obtain an accurate answer. However, it could be hypothesized that the sample number employed was small, and also not equal. Another limitation of this work is related to the questionnaire, that although it has a clear delimitation about the activities that correspond to each level, it is in the end the participants (through their self-report) who indicated if the activity fell in one or another category or aerobic level. This could generate difficulties at the moment of the METs calculation. Besides, it is important to highlight that the number of women and men in each group was not equal, however no significant differences were found about this fact. As it was mentioned in the introduction, it was found that the hippocampus is more developed in those subjects who had a better cardiorespiratory level because of their aerobic training, indicating the relevance of maintaining an active style of life. Therefore, the current evidence suggests that performing physical activity could be a promising intervention to reduce the cognitive impairment which occurs as a person gets older. Promoted from the early childhood and sustained throughout adulthood, in each of the life-stages, physical activity could be positioned as an appealing intervention, which could be easily performed for any type of population and in any environment. 
